\title{
Evaluatie van de mini-FLOTAC-methode voor de detectie van gastro-intestinale parasieten bij grote huisdieren
}

\author{
Evaluation of the mini-FLOTAC technique for detection of \\ gastro-intestinal parasites in large companion animals
}

\author{
N. Van den Putte, E. Claerebout, B. Levecke
}

Vakgroep Virologie, Parasitologie en Immunologie

Faculteit Diergeneeskunde, Universiteit Gent, Salisburylaan 133, 9820 Merelbeke, België

Niels.VandenPutte@UGent.be

\section{AMENVATTING}

Voor de microscopische diagnose van gastro-intestinale parasieten bij grote huisdieren werd recent een nieuwe techniek ontwikkeld, de mini-FLOTAC. In deze vergelijkende studie van diagnostische technieken werd de mini-FLOTAC vergeleken met de standaard McMastertechniek op basis van de detectie (gevoeligheid) en kwantificatie (aantal oöcysten/eieren per gram mest (OPG/EPG)) van parasitaire infecties bij grote huisdieren en de tijd die nodig is om een diagnose te stellen. In totaal werden honderd meststalen (78 paarden, 11 runderen, 6 schapen en 5 geiten) onderzocht met beide technieken. Mini-FLOTAC was gevoeliger dan McMaster, maar er was een zeer hoge correlatie in eieren per gram (EPG) feces (correlatiecoëfficiënt $=0,90, p<$ $0,001)$. De benodigde tijdsduur voor het onderzoeken van stalen met de mini-FLOTAC-techniek (13 minuten) lag significant hoger $(p<0,05)$ dan de tijd die nodig was met de McMaster-techniek (6 minuten). Dit verschil in tijdsduur was eveneens afhankelijk van de bekomen EPG-waarde, waarbij het tijdsverschil tussen beide technieken nog sterk vergrootte voor hogere waarden. Er kan geconcludeerd worden dat de mini-FLOTAC-techniek het beste gebruikt kan worden wanneer een accurate diagnose van een lage besmettingsgraad nodig is, bijvoorbeeld voor de detectie van anthelminthicumresistentie. In andere gevallen kan de voorkeur worden gegeven aan de snellere McMaster-methode.

\begin{abstract}
A new technique, mini-FLOTAC, has recently been developed for the microscopic diagnosis of infections with gastro-intestinal nematodes in domestic animals. In this comparative study of diagnostic techniques, the mini-FLOTAC technique was compared to the commonly used McMaster technique for the detection and quantification of gastro-intestinal parasites in livestock as well as for the evaluation of the time needed to perform the assays. One hundred fecal samples ( 78 horses, 11 bovines, 6 sheep and 5 goats) were examined with both the mini-FLOTAC and McMaster technique. This revealed that more infections with gastro-intestinal parasites could be diagnosed with the mini-FLOTAC technique than with the McMaster technique, but the comparative study also showed a very high correlation (correlation coefficient $=0.90, \mathrm{p}<0.001$ ) between both techniques regarding the number of eggs per gram feces (EPG) detected. The time needed to examine the samples with the mini-FLOTAC technique $(13$ minutes $)$ was significantly higher $(\mathrm{P}<0.05)$ than the time needed with the McMaster technique (6 minutes). This difference in time also depended on the observed EPG value, as with high EPG values, the difference in time between both techniques increased even more. In conclusion, the mini-FLOTAC technique can be preferred when an accurate diagnosis of a low infection level is necessary, e.g. to detect anthelmintic resistance. In other circumstances, the faster McMaster technique can be considered as the preferable technique.
\end{abstract}




\section{INLEIDING}

Besmettingen met maagdarmparasieten zijn veel voorkomend bij grote huisdieren. Deze besmettingen hebben een invloed op zowel de gezondheid als de productiecapaciteit van deze dieren en worden vooral bestreden door middel van antiparasitaire behandelingen (Morgan et al. 2005). Deze infecties worden momenteel voornamelijk gediagnosticeerd aan de hand van coprologisch onderzoek. Dit onderzoek dient kwantitatief te gebeuren omdat de meeste grote huisdieren besmet zijn met een laag aantal maagdarmparasieten en deze subklinische besmettingen vaak geen economische impact hebben. Deze lage besmettingen zijn wel belangrijk in het kader van anthelminthicumresistentie. Wanneer ze kort na een antiparasitaire behandeling worden gediagnosticeerd, kunnen ze namelijk wijzen op een beginnende resistentie tegen een bepaald anthelminthicum (Rinaldi et al., 2014).

De McMaster-techniek wordt momenteel het frequentst aangewend voor deze kwantitatieve diagnostiek. De nadelen van deze techniek zijn echter haar lage gevoeligheid, waardoor een lage besmettingsgraad kan worden gemist, en de lage precisie waarmee de eitellingen worden uitgevoerd (Rinaldi et al., 2014). Om aan deze lage gevoeligheid te verhelpen werd door Cringoli et al. (2012) de mini-FLOTACtechniek ontwikkeld. Deze nieuwe techniek is afgeleid van de FLOTAC-techniek, maar is gebruiksvriendelijker, omdat er geen centrifugatie nodig is. Bovendien heeft ze een hogere gevoeligheid dan de McMaster-techniek (Cringoli et al., 2010).

In deze studie wordt een vergelijking gemaakt tussen twee diagnostische technieken, de McMaster- en mini-FLOTAC-techniek, en dit zowel op basis van de gevoeligheid van beide technieken als op basis van de tijdsduur nodig om beide uit te voeren. Op basis van de resultaten van deze vergelijking kan vervolgens een aanbeveling tot gebruik van beide technieken geformuleerd worden.

\section{MATERIALEN EN METHODEN}

\section{Meststalen}

Voor deze studie werd gebruik gemaakt van meststalen van grote huisdieren (paarden, runderen, geiten en schapen) aangeboden aan het Laboratorium van Parasitologie van de Faculteit Diergeneeskunde te Merelbeke (UGent) tussen september 2014 en maart 2015. Deze stalen werden doorgestuurd door dierenartsen zowel binnen (kliniek Inwendige Ziekten van de Grote Huisdieren) als buiten de Faculteit Diergeneeskunde. In totaal werden honderd individuele meststalen onderzocht, waarvan 78 paarden, 11 runderen, 6 schapen en 5 geiten. Deze staalnamegrootte is gebaseerd op het aantal stalen dat binnen voorgenoemde studieperiode individueel kon worden onderzocht.

\section{Parasitologisch onderzoek}

Ieder staal werd op dezelfde dag onderzocht aan de hand van de McMaster- en de mini-FLOTAC-techniek door eenzelfde persoon. Aanvullend werd ook de tijdsduur nagegaan voor het maken van een diagnose voor ieder van de twee technieken.

\section{McMaster}

De McMaster werd uitgevoerd zoals beschreven door El-Abdellati (2010) en kan worden opgesplitst in vijf opeenvolgende stappen. Er werd eerst $4 \mathrm{~g}$ mest afgewogen (stap 1). Vervolgens werd deze hoeveelheid mest vermengd met $60 \mathrm{ml}$ verzadigde zoutoplossing met een specifieke densiteit van $1,24(333 \mathrm{~g} \mathrm{NaCl}$ en $200 \mathrm{~g}$ sucrose in 1 liter gedistilleerd water). De suspensie werd driemaal door een theezeef gezeefd om het meeste debris te verwijderen. Vóór het vullen van iedere cel werd de suspensie gehomogeniseerd door ze tienmaal over te gieten van de ene beker in een andere. Daarna werden de twee cellen van een McMaster-telkamer gevuld met deze suspensie (stap 2). Na een periode van twee minuten (stap 3 ) werd het aantal oöcysten/eieren in iedere cel geteld $(2 \times 0,15 \mathrm{ml})$ aan de hand van een lichtmicroscoop (100X vergroting) (stap 4). Finaal werd al het gebruikte materiaal gewassen, zodat een nieuw meststaal onderzocht kon worden (stap 5). Het aantal oöcysten/eieren per gram mest (OPG/EPG) werd bekomen door het getelde aantal oöcysten/eieren te vermenigvuldigen met 50 . De benodigde tijd voor ieder van de vijf stappen werd afzonderlijk gemeten voor elk van de meststalen. Een visuele tutorial om een meststaal te onderzoeken met de McMaster-techniek kan worden gevonden op www.youtube.com/watch?v=rkSGe-L4Sec.

\section{Mini-FLOTAC}

De mini-FLOTAC werd uitgevoerd zoals beschreven in de handleiding voor herbivoren (persoonlijke communicatie G. Cringoli, 2014), en bestaat uit zes opeenvolgende stappen. Allereerst werd $5 \mathrm{~g}$ mest afgewogen in de fill-FLOTAC (stap 1) (Figuur 1). Hieraan werd vervolgens $45 \mathrm{ml}$ verzadigde zoutoplossing (cf. McMaster-techniek) toegevoegd. Na het sluiten van de fill-FLOTAC werd de mest in suspensie gebracht door een op- en neerwaartse beweging van het mengstaafje. Vervolgens werden beide cellen van de Mini-FLOTAC-telkamer (Figuur 1) gevuld onder een hoek van ongeveer $25-30^{\circ}$, na het homogeniseren van de suspensie (10 op- en neerwaartse bewegingen met mengstaafje) vóór het vullen van iedere cel (stap 2). Na een incubatieperiode van tien minuten (stap 3) werd de bovenste laag van de suspensie (met de oöcysten en eieren) in de mini-FLOTAC gescheiden van de onderste laag door een draaibeweging met de sleutel ('translatie') (stap 4). De oöcysten en eieren werden geteld aan de hand van een lichtmicroscoop 


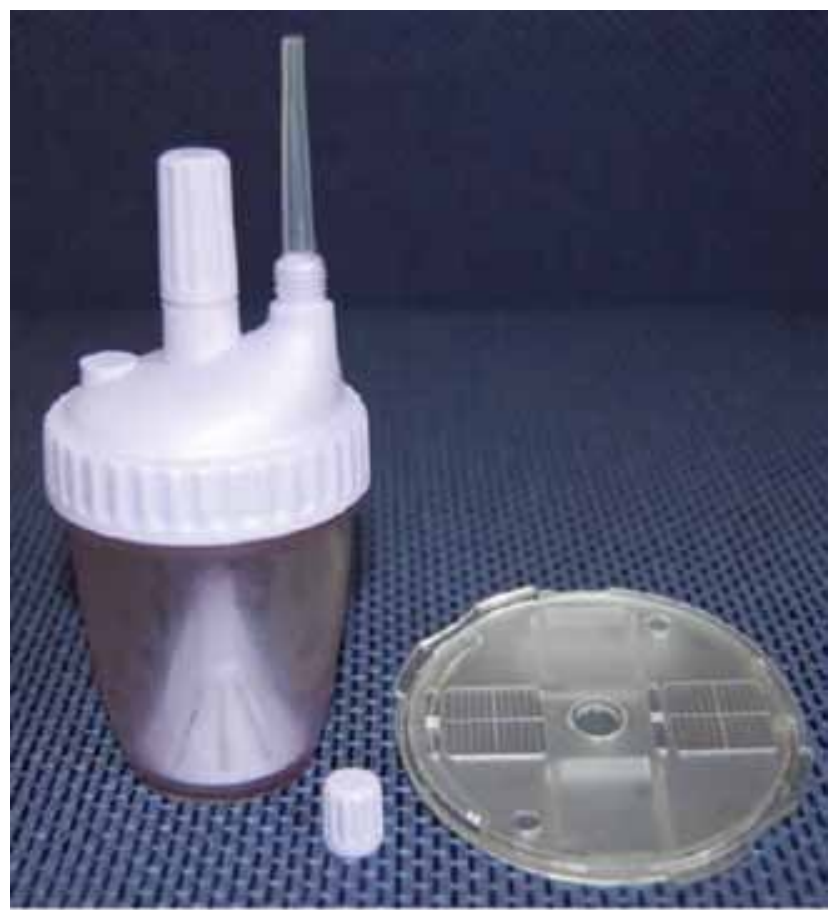

\section{Fill - FLOTAC Mini - FLOTAC}

Figuur 1. Links: Fill-FLOTAC; Rechts: mini-FLOTACtelkamer. (Bron: Barda et al., 2013)

(100X vergroting) (stap 5). Finaal werd al het gebruikte materiaal gewassen zodat een nieuw meststaal onderzocht kon worden (stap 6). Het aantal oöcysten/ eieren per gram mest (OPG/EPG) werd bekomen door de getelde oöcysten/eieren te vermenigvuldigen met 5 . De benodigde tijd voor ieder van de zes stappen werd afzonderlijk gemeten voor elk van de meststalen. Een visuele tutorial om een meststaal te onderzoeken met de mini-FLOTAC-techniek kan worden gevonden op https://www.youtube.com/watch?v=65Ch9PZvfxI.

\section{STATISTISCHE ANALYSE}

Beide technieken werden vergeleken op basis van de gevoeligheid, de oöcyst/eitellingen en de tijd nodig om een meststaal te verwerken. Omdat er geen perfecte standaardtechniek bestaat voor de diagnose van parasitaire infecties (gevoeligheid $=100 \%$ en specificiteit $=100 \%$ ), werd er aangenomen dat een dier geparasiteerd was wanneer met minstens één van de twee technieken oöcysten of eieren werden gevonden ( specificiteit $=100 \%$ ). De sensitiviteit voor beide technieken werd bepaald voor de diagnose van een parasitaire infectie, ongeacht de soort parasiet, en voor elke parasietensoort afzonderlijk. Indien er een voldoende aantal dieren geïnfecteerd was $(n>50)$, werd ook de impact van de intensiteit van infectie op de gevoeligheid nagegaan. Hiervoor werd de sensitiviteit bepaald over verschillende niveaus van oöcyst/ ei-uitscheiding (1-50 OPG/EPG; 51-99 OPG/EPG;
100-149 OPG/EPG; $\geq 150$ OPG/EPG), waarbij de oöcyst/ei-uitscheiding gelijk was aan de gemiddelde oöcyst/eitelling van de twee technieken. De oöcyst/ eitelling bekomen met McMaster en mini-FLOTAC per parasiet werd vergeleken aan de hand van de spearmann-correlatiecoëfficiënt wanneer minstens vijftig dieren geparasiteerd waren. Verschillen in oöcyst/eitelling werden nagegaan met een tweezijdige, gepaarde t-test.

De gemiddelde tijd nodig om een staal te verwerken werd bepaald voor beide technieken. Een significant verschil in tijd werd vervolgens nagegaan aan de hand van een gepaarde t-test. Omdat het tellen van oöcysten/eieren in de kamers een invloed heeft op de afleestijd en dit effect niet noodzakelijk voor de twee technieken even groot is, werd de correlatie tussen tijd en het totaal aantal parasieten (som van het aantal getelde oöcysten en eieren) voor beide technieken afzonderlijk bepaald. Deze relatie tussen het totaal aantal getelde parasieteneieren/oöcysten en tijd werd nagegaan aan de hand van een lineaire regressie met tijd (in seconden) als de afhankelijke variabele en twee onafhankelijke variabelen op basis van het totaal aantal parasieteneieren/oöcysten geteld onder de microscoop: de ruwe telling en de log getransformeerde telling.

Finaal werd op basis van de bekomen resultaten bepaald wat de meest efficiënte manier was om parasitaire infecties op te sporen bij grote huisdieren. Hiervoor werd de tijd per diagnose berekend voor de twee technieken ( $=$ tijd voor het onderzoeken van alle stalen / totaal van diagnosen). Er werd nagegaan of het onderzoeken van stalen in serie (eerst het onderzoeken van alle stalen met de McMaster-techniek en vervolgens de negatieve stalen met de mini-FLOTACtechniek) een mogelijk alternatief is voor het opsporen van alle infecties.

\section{RESULTATEN}

Een totaal van zes verschillende gastro-intestinale parasieten werden gevonden in de honderd meststalen. De meest voorkomende parasieten waren strongyliden (56/100). In een minderheid van de stalen werd Parascaris equorum (5/100), Eimeria spp. (6/100), Anoplocephala spp. (3/100), Nematodirus spp. $(2 / 100)$ en Trichuris spp. $(2 / 100)$ gevonden. In Tabel 1 wordt de distributie van deze parasitaire infecties over de diersoorten heen beschreven voor de twee technieken afzonderlijk.

\section{Gevoeligheid}

Mini-FLOTAC was het meest gevoelig. De techniek detecteerde 73 van de 74 parasitaire infecties (98,6\%), waar McMaster 59 (79,7\%) van deze infecties opspoorde. Strongylideninfecties werden in 55 van de 56 gevallen $(98,2 \%)$ opgespoord door mini- 
Tabel 1. Het aantal positieve stalen met de McMaster-, de mini-FLOTAC-techniek en een combinatie van beide voor vier soorten grote huisdieren.

\begin{tabular}{|c|c|c|c|c|}
\hline \multirow[t]{2}{*}{ Diersoort } & \multirow[t]{2}{*}{ Parasiet } & \multirow[b]{2}{*}{ Combinatie van technieken } & \multicolumn{2}{|c|}{ Aantal positieve stalen } \\
\hline & & & McMaster & Mini-FLOTAC \\
\hline \multirow[t]{3}{*}{ Paard $(n=78)$} & Strongyliden & 44 & 35 & 43 \\
\hline & Parascaris equorum & 5 & 4 & 5 \\
\hline & Anoplocephala spp. & 3 & 1 & 3 \\
\hline \multirow[t]{3}{*}{ Rund (n= 11) } & Strongyliden & 4 & 3 & 4 \\
\hline & Nematodirus spp. & 1 & 1 & 1 \\
\hline & Eimeria spp. & 4 & 4 & 4 \\
\hline \multirow[t]{3}{*}{ Schaap $(n=6)$} & Strongyliden & 3 & 3 & 3 \\
\hline & Trichuris spp. & 2 & 1 & 2 \\
\hline & Eimeria spp. & 1 & 1 & 1 \\
\hline \multirow[t]{3}{*}{ Geit $(n=5)$} & Strongyliden & 5 & 5 & 5 \\
\hline & Nematodirus spp. & 1 & 0 & 1 \\
\hline & Eimeria spp. & 1 & 1 & 1 \\
\hline Totaal & & 74 & 59 & 73 \\
\hline
\end{tabular}

Tabel 2. De gevoeligheid van McMaster voor de detectie van strongylideninfecties voor vier niveaus van uitscheiding.

\begin{tabular}{lcccc}
\hline & \multicolumn{5}{c}{ Gemiddelde ei-uitscheiding } \\
\hline & $1-49$ & $50-99$ & $100-149$ & $\geq 150$ \\
\hline Gevoeligheid McMaster & $\begin{array}{c}43,8 \% \\
7 / 16\end{array}$ & $\begin{array}{c}91,7 \% \\
11 / 12\end{array}$ & $\begin{array}{c}100 \% \\
4 / 4\end{array}$ & $\begin{array}{c}100 \% \\
2 / 24\end{array}$ \\
\hline
\end{tabular}

FLOTAC en in $46(82,1 \%)$ van de gevallen door McMaster. In Tabel 2 wordt aangetoond dat de sensitiviteit van McMaster varieert over verschillende niveaus van uitscheiding van strongyliden. McMaster faalde om lage uitscheiders op te sporen, maar had een gevoeligheid van $100 \%$ wanneer de ei-uitscheiding minstens 100 EPG was.

\section{Ei-uitscheiding}

Figuur 2 illustreert de overeenkomst tussen ei-uitscheiding geschat door McMaster en mini-FLOTAC. Er was een significant positieve correlatie tussen de eiuitscheiding geschat door beide technieken (correlatiecoëfficiënt $=0,90, \mathrm{p}<0,001)$. Wanneer de stalen werden onderzocht met de McMaster-techniek was de gemiddelde uitscheiding 185,5 EPG; op basis van de miniFLOTAC-techniek was dit 201,1 EPG. Er was geen significant verschil in ei-uitscheiding tussen beide technieken (gemiddelde verschil $=-15,6$ EPG 95\% betrouwbaarheidsinterval [-7,75 EPG tot 38,9 EPG], $p=0,19)$.

\section{Tijdsduur van de analyses}

Het verwerken van een meststaal met mini-FLOTAC (13 min $23 \mathrm{sec}$ ("standard error of mean" (SEM) 36 sec)) duurde twee keer zolang als bij de McMaster- techniek (6 min $35 \mathrm{sec}$ (SEM: $12 \mathrm{sec})$ ). Deze verhouding nam af wanneer rekening gehouden werd met het aantal diagnosen. De gemiddelde tijd voor een diagnose (totale tijd / aantal diagnosen) was $18 \mathrm{~min}$ $21 \mathrm{sec}$ voor mini-FLOTAC (22 u $19 \mathrm{~min} 35 \mathrm{sec} / 73$ diagnosen) en $11 \mathrm{~min} 10 \mathrm{sec}(10 \mathrm{u} 58 \mathrm{~min} 52 \mathrm{sec} / 59$ diagnosen) voor McMaster, resulterende in een verhouding van 1,5 .

De verschillen in tijd tussen beide technieken was

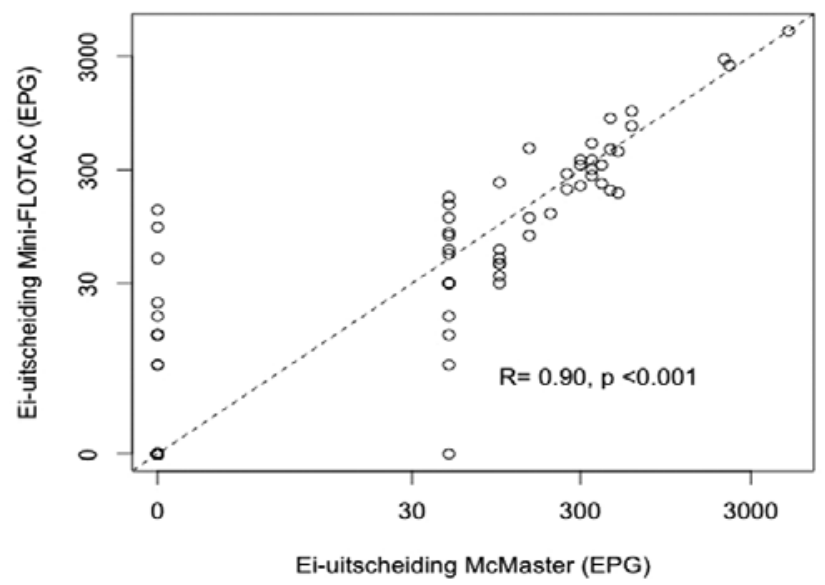

Figuur 2. Correlatie tussen EPG-waarden bekomen met de mini-FLOTAC-techniek (Y-as) en de McMastertechniek (X-as). 


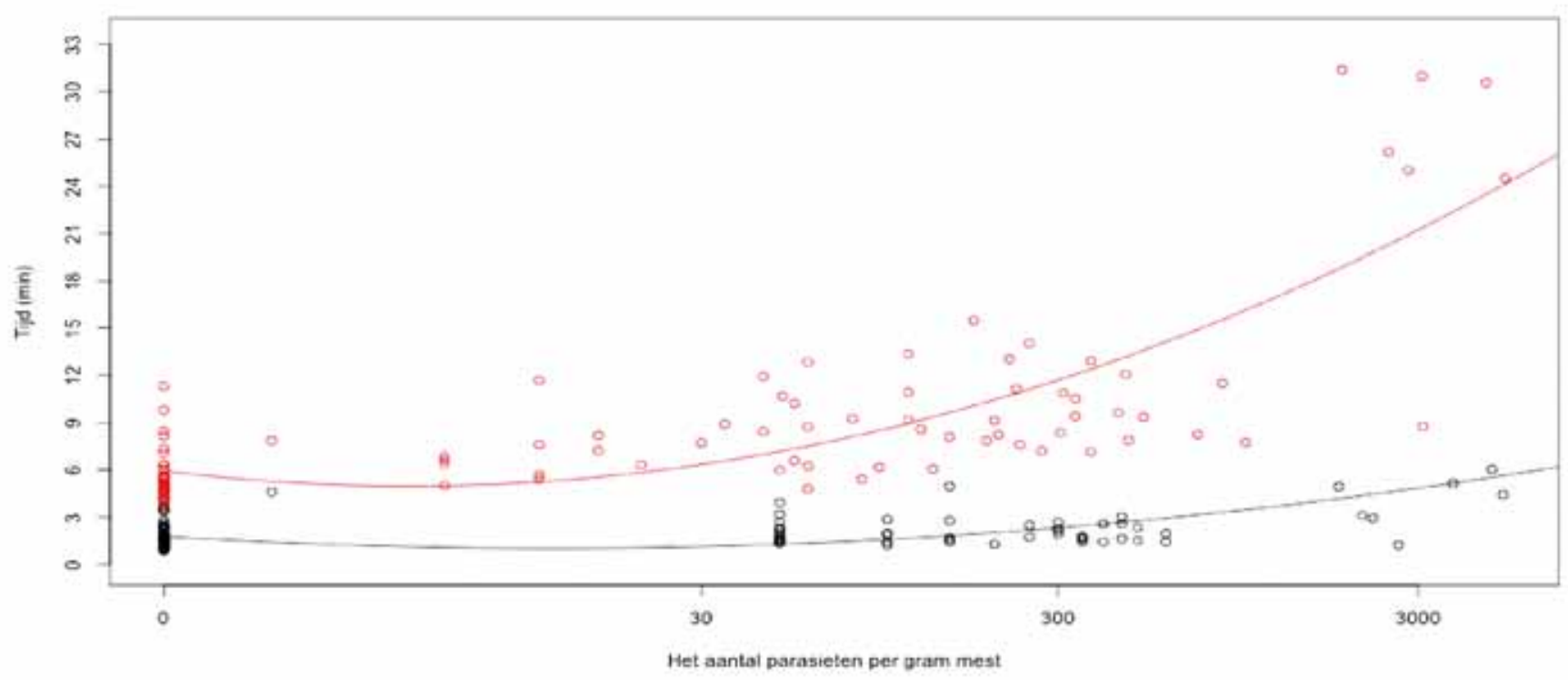

Figuur 3. Tijd nodig voor het aflezen van een staal in verhouding tot het aantal parasietdeeltjes per gram mest. Rood: mini-FLOTAC; zwart: McMaster.

voornamelijk te wijten aan de tijd nodig voor het microscopisch onderzoeken van de telkamers (McMaster: 2 min $10 \mathrm{sec}$ versus mini-FLOTAC: 8 min 34 $\mathrm{sec}$ ) en het wassen van de gebruikte materialen (McMaster: 0 min $37 \mathrm{sec}$ versus mini-FLOTAC: 1 min 28 sec) (Tabel 3). Figuur 3 illustreert ook de toename in de tijd die nodig is voor het microscopisch onderzoeken van de telkamers in functie van het totaal aantal eieren/oöcysten per gram mest. Voor beide technieken was er een significante toename in tijd wanneer het aantal eieren/oöcysten toenam, maar deze toename was niet lineair en was groter voor mini-FLOTAC.

In Tabel 4 worden de totale tijd nodig om honderd stalen microscopisch te onderzoeken, het aantal diag- nosen en de tijd per diagnose voor verschillende diagnostische strategieën beschreven. De verschillende diagnostische strategieën die gebruikt werden waren: (i) Slechts één techniek om alle stalen te onderzoeken, ofwel McMaster ofwel mini-FLOTAC, (ii) beide technieken in serie, waarbij eerst de ene techniek werd toegepast op alle stalen en vervolgens de andere op de stalen die negatief testten, (iii) beide technieken in parallel, waarbij elk staal met elk van beide technieken werd onderzocht.

Algemeen was er een toename in diagnosen wanneer de technieken werden gecombineerd, maar de mate van toename varieerde tussen de technieken en hoe ze werden gecombineerd (in serie versus in parallel). De

Tabel 3. Gemiddelden met standaardfout van de tijd nodig voor elke stap bij beide technieken.

\begin{tabular}{lll}
\hline & McMaster & Mini-FLOTAC \\
\hline Wegen & $0 \min 44 \mathrm{sec}+/-1,91 \mathrm{sec}$ & $0 \mathrm{~min} 48 \mathrm{sec}+/-1,85 \mathrm{sec}$ \\
Klaarmaken & $3 \mathrm{~min} 04 \mathrm{sec}+/-2,88 \mathrm{sec}^{*}$ & $2 \mathrm{~min} 24 \mathrm{sec}+/-2,53 \mathrm{sec}^{*}$ \\
Aflezen (alle stalen) & $2 \min 10 \mathrm{sec}+/-9,02 \mathrm{sec}^{*}$ & $8 \mathrm{~min} 44 \mathrm{sec}+/-33,68 \mathrm{sec}^{*}$ \\
Afwassen & $0 \min 37 \mathrm{sec}+/-1,43 \mathrm{sec}^{*}$ & $1 \mathrm{~min} 28 \mathrm{sec}+/-2,32 \mathrm{sec}^{*}$ \\
\hline Totaal & $6 \min 35 \mathrm{sec}+/-12,06 \mathrm{sec}^{*}$ & $13 \mathrm{~min} 24 \mathrm{sec}+/-36,31 \mathrm{sec}^{*}$ \\
\hline
\end{tabular}

$* \mathrm{P}<0,05$

Tabel 4. Totale tijdsduur, aantal diagnosen en tijd per diagnose voor vier diagnostische strategieën.

\begin{tabular}{|c|c|c|c|}
\hline Strategie & Totale tijdsduur & Aantal diagnosen & Tijd per diagnose \\
\hline $\mathrm{McM}$ & 10 u $58 \mathrm{~min} 52 \mathrm{sec}$ & 59 & $11 \mathrm{~min} 10 \mathrm{sec}$ \\
\hline $\operatorname{McM}(1)+\operatorname{MF}(2)$ & 19 u $22 \min 5 \mathrm{sec}$ & 71 & $16 \mathrm{~min} 23 \mathrm{sec}$ \\
\hline MF & 22 u $19 \min 33 \mathrm{sec}$ & 73 & $18 \min 21 \mathrm{sec}$ \\
\hline $\mathrm{MF}(1)+\mathrm{McM}(2)$ & $26 \mathrm{u} 01 \mathrm{~min} 35 \mathrm{sec}$ & 74 & $21 \mathrm{~min} 06 \mathrm{sec}$ \\
\hline $\mathrm{McM}+\mathrm{MF}$ & $33 \mathrm{u} 18 \mathrm{~min} 27 \mathrm{sec}$ & 74 & $29 \mathrm{~min} 31 \mathrm{sec}$ \\
\hline
\end{tabular}

McM: McMaster; MF: mini-FLOTAC 


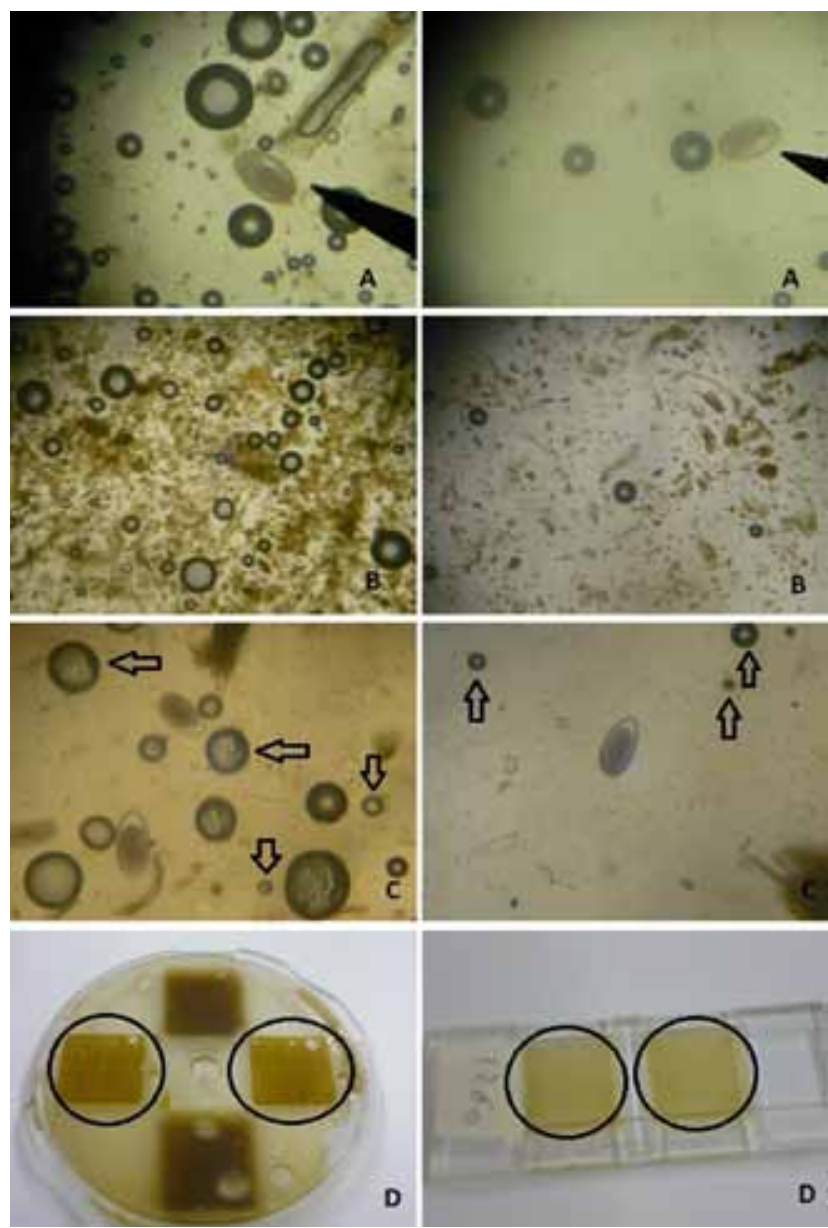

Figuur 4. Verschillen tussen beide technieken bij het aflezen onder de microscoop. Links: Mini-FLOTAC; Rechts: McMaster; A. Klaarheid, B. Vuildeeltjes, C. Microscopische luchtbellen, D. Macroscopische luchtbellen.

grootste toename werd waargenomen voor McMaster, waarvoor het aantal diagnosen steeg van 59 (enkel McMaster) tot meer dan 71 (McMaster gecombineerd met mini-FLOTAC). Voor mini-FLOTAC was het aantal diagnosen minstens 71 , ongeacht de diagnostische strategie. Het verschil in aantal diagnosen tussen het onderzoeken van meststalen in parallel $(\mathrm{n}=$ 74) en in serie (McMaster-mini-FLOTAC $=71$; miniFLOTAC-McMaster $=74$ ) was klein. De tijd per diagnose daarentegen varieerde van $16 \min 23 \mathrm{sec}$ voor McMaster-mini-FLOTAC (serie), over $21 \mathrm{~min} 06 \mathrm{sec}$ voor mini-FLOTAC-McMaster (serie) tot $27 \mathrm{~min} 00$ sec wanneer beide technieken in parallel werden toegepast.

\section{BESPREKING}

In deze studie werd de mini-FLOTAC-techniek vergeleken met de McMaster voor de diagnose van gastro-intestinale parasieten bij grote huisdieren (paarden, runderen, schapen en geiten) op basis van (i) gevoeligheid, (ii) eitellingen en (iii) de tijd nodig voor het verwerken van de stalen.

De resultaten tonen aan dat mini-FLOTAC gevoeliger is dan McMaster (over de parasietsoorten heen: $98,6 \%$ versus $79,7 \%$, strongyliden: $98,2 \%$ versus $82,1 \%$ ), en bevestigen de conclusies van recente studies die beide technieken valideerden voor de diagnose van gastro-intestinale parasieten bij schapen (strongyliden: $100 \%$ versus $75,9-88,5 \%$ ) (Rinaldi et al., 2014) en mensen (Hymenolepis nana: 77,8-92,7\% versus $60,5 \%$; Ascaris lumbricoides: $61,3-87,1 \%$ versus 48,3\%) (Barda et al., 2014). Zoals beschreven voor strongylideninfecties bij schapen (Rinaldi et al., 2014) faalde McMaster ook om lage ei-uitscheiders op te sporen bij paarden en runderen, maar werd de techniek $100 \%$ gevoelig van zodra de ei-uitscheiding minstens 100 EPG was. Dit verschil in gevoeligheid tussen de technieken in functie van de intensiteit van ei-uitscheiding is niet onverwacht en kan verklaard worden door een verschil in de hoeveelheid mest die wordt onderzocht $(0,1$ gram voor mini-FLOTAC versus 0,02 gram voor McMaster). Hoe meer mest er wordt onderzocht, hoe groter de kans op het detecteren van een ei/oöcyst, maar de minimale hoeveelheid mest die moet onderzocht worden voor het opsporen van één ei/oöcyst is kleiner als het aantal uitgescheiden eieren/oöcysten in 1 gram mest groot is (Levecke et al., 2011; Torgerson et al., 2012). Ondanks deze significante verschillen in gevoeligheid resulteerden beide technieken in vergelijkbare schattingen van de ei-uitscheiding. Hoewel dit te wijten kan zijn aan een te kleine steekproefgrootte, werd de afwezigheid van een significant verschil in ei-uitscheiding tussen technieken met een verschillende gevoeligheid reeds eerder beschreven (Levecke et al., 2009; 2011; 2015; Rinaldi et al., 2014).

De relatie tussen gevoeligheid en ei-uitscheiding, en de afwezigheid van significante verschillen in eiuitscheiding tussen technieken met een verschillende gevoeligheid krijgen tot op heden onvoldoende aandacht. Toch hebben beide observaties belangrijke consequenties voor de keuze van de diagnostische methode voor het opsporen van gastro-intestinale parasieten. Allereerst gaat een verhoogde gevoeligheid vaak gepaard met een verhoogde kost van de diagnose (Levecke et al., 2009; Speich et al., 2010; Barda et el., 2014). Dit werd ook bevestigd in de voorliggende studie door de grote verschillen tussen beide technieken wat betreft de tijd die nodig is voor het verwerken van een staal (mini-FLOTAC: 13 min $23 \mathrm{sec}$ versus McMaster: 6 min $35 \mathrm{sec})$. Ten tweede hebben infecties met een lage intensiteit ( $\sim$ ei-uitscheiding) weinig of geen klinisch belang. In Nederland bijvoorbeeld wordt het afgeraden om paarden die minder dan 500 EPG uitscheiden, te behandelen (www.parasietenwijzer.nl). Dit is een niveau van ei-uitscheiding dat, op basis van de bekomen data, met hoge waarschijnlijkheid ook met de McMaster-techniek zal worden opgespoord en accuraat zal worden geschat, maar wel 
dubbel zo snel als wanneer de mini-FLOTAC zou gebruikt worden. Indien toch een maximale gevoeligheid wordt nagestreefd voor een minimale kost is het aan te raden om eerst de stalen te onderzoeken met de McMaster, om daarna alle stalen waarin geen parasieten werden gevonden opnieuw te onderzoeken met de mini-FLOTAC-techniek. Deze strategie vraagt 1,76 keer meer tijd dan wanneer McMaster alleen wordt gebruikt, maar verhoogt de sensitiviteit van $79,7 \%$ naar $95,9 \%$. In vergelijking met het exclusieve gebruik van mini-FLOTAC, daalt de sensitiviteit van $98,6 \%$ naar $95,9 \%$, maar worden de diagnosen 0,74 keer sneller gemaakt.

Dit is de eerste studie waarin de tijd afzonderlijk werd geëvalueerd voor zowel McMaster en miniFLOTAC. De resultaten tonen aan dat stalen sneller kunnen klaargemaakt worden voor de mini-FLOTAC ( 2 min $24 \mathrm{sec}$ versus $3 \mathrm{~min} 04 \mathrm{sec}$ ), maar dat meer tijd nodig is voor het aflezen ( $8 \mathrm{~min} 44 \mathrm{sec}$ versus $2 \mathrm{~min}$ $10 \mathrm{sec}$ ) en het wassen van de gebruikte materialen (1 min $28 \mathrm{sec}$ versus $37 \mathrm{sec}$ ). Het gebruik van de fillFLOTAC verklaart zowel het sneller klaarmaken als het trager reinigen van de gebruikte materialen: het toestelletje combineert drie verschillende stappen bij het verwerken van de stalen (i.e. het homogeniseren, zeven en overbrengen van de suspensie in de telkamers), maar is moeilijker te reinigen. Het verschil in tijd voor het aflezen van de telkamers kan verklaard worden door een verschil in (i) oppervlakte van de telkamers (mini-FLOTAC: $648 \mathrm{~mm}^{2}$ versus McMaster: $200 \mathrm{~mm}^{2}$ ), (ii) de hoeveelheid onderzochte mest, en dus het aantal eieren dat geteld moet worden (voor ieder ei dat geteld wordt met McMaster moeten er tien geteld worden met mini-FLOTAC), (iii) de hoeveelheid artefacten (meer aanwezig bij mini-FLOTAC) (Figuur 4 A, B, C, D) en het aantal microscopische luchtbellen (meer aanwezig bij mini-FLOTAC) (Figuur $4 \mathrm{~A}, \mathrm{~B}, \mathrm{C}, \mathrm{D})$. Bij mini-FLOTAC waren soms ook macroscopisch zichtbare luchtbellen aanwezig. Dit is hoogstwaarschijnlijk te wijten aan slijtage van de onderdelen van de mini-FLOTAC-telkamers.

Deze studie heeft echter enkele tekortkomingen. Allereerst kunnen besluiten over de diagnostische waarde van beide technieken enkel genomen worden voor strongylideninfecties bij paarden (aantal geïnfecteerde dieren $=44$ ). Voor de andere parasieten en diersoorten was het aantal geïnfecteerde dieren te klein $(\leq 5)$ voor een sluitende analyse. Ten tweede werd de gevoeligheid bepaald in afwezigheid van een perfecte standaardtechniek. Hoewel een combinatie van de testresultaten van beide technieken de kans op een valsnegatief testresultaat verkleint, kan dit niet $100 \%$ worden uitgesloten. De gerapporteerde resultaten betreffende de gevoeligheid zijn daarom een onderschatting. Als alternatief wordt vaak gebruik gemaakt van 'latent class'-modellen in een Bayesiaans kader (Hui en Zhou 1998; Enøe et al., 2000; Berkvens et al., 2006), waarbij vooraf informatie over de gevoeligheid van de technieken op basis van een expertopinie kan worden ingevoegd. Deze modellen houden echter tot op heden geen rekening met de variatie in gevoeligheid voor ei-uitscheiding (Levecke et al., 2015). Ten derde werd deze studie uitgevoerd door één persoon in één laboratorium, waardoor extrapolatie van de resultaten zowel binnen het Laboratorium voor $\mathrm{Pa}$ rasitologie van de Faculteit Diergeneeksunde (UGent) als naar andere laboratoria met enige voorzichtigheid moet gebeuren. Hoewel dit in het bijzonder van toepassing is voor de tijd die nodig is voor het verwerken van de stalen, zijn de tijdsopnamen voor McMaster en mini-FLOTAC vergelijkbaar met deze gerapporteerd in andere studies bij mensen (McMaster: $7 \mathrm{~min} / \mathrm{staal}$; mini-FLOTAC: $13 \mathrm{~min} / \mathrm{staal}$ (Barda et al., 2014)).

\section{CONCLUSIE}

In de studie wordt aangetoond dat mini-FLOTAC gevoeliger is dan de huidige McMaster-methode, in het bijzonder voor de diagnose van infecties met een lage intensiteit en dat eitellingen tussen de technieken vergelijkbaar zijn, maar dat de mini-FLOTACtechniek meer tijd vraagt om uit te voeren. Rekening houdend met deze meerkost voor de diagnose en de minimale meerwaarde van mini-FLOTAC voor de diagnose van infecties met een hoge intensiteit en van klinisch belang zijn, blijft het gebruik van McMaster verantwoord. Indien toch een maximale gevoeligheid wordt nagestreefd voor een minimale kost (bijvoorbeeld voor de detectie van anthelminthicumresistentie) is het aan te raden om beide technieken in serie uit te voeren, waarbij eerst de McMaster-techniek wordt toegepast op alle stalen en vervolgens de miniFLOTAC op de stalen die negatief testten.

\section{REFERENTIES}

Barda B., Cajal P., Villagran E., Cimino R., Juarez M., Krolewiecki A., Rinaldi L., Cringoli G., Burioni R., Albonico M. (2014). Mini-FLOTAC, Kato-Katz and McMaster: three methods, one goal; highlights from north Argentina. Parasites \& Vectors 7, 271.

Barda B.D., Rinaldi L., Ianniello D., Zepherine H., Salvo F., Sadutshang T., Cringoli G., Clementi M., Albonico M. (2013). Mini-FLOTAC, an innovative direct diagnostic technique for intestinal parasitic infections: experience from the field. PLoS Neglected Tropical Diseases 7(8), e2344.

Berkvens D., Speybroeck N., Praet N., Adel A., Lesaffre E. (2006). Estimating disease prevalence in a Bayesian framework using probabilistic constraints. Epidemiology 17, 145-153.

Cringoli G., Maurelli M.P., Pepe P., Alfano S., Pennacchio S., Ianniello D., Rinaldi L. (2012). Mini-FLOTAC, a new tool for copromicroscopic diagnosis of Toxocara canis, Ancylostoma caninum and Trichuris vulpis in dogs. In: Proceedings of the XXVII Congress of the Italian Society of Parasitology (SOIPA). Alghero, 26-29 June 2012, 197.

Cringoli G., Rinaldi L., Maurelli M.P., Utzinger J. (2010). 
FLOTAC: new multivalent techniques for qualitative and quantitative copromicroscopic diagnosis of parasites in animals and humans. Nature Protocols 5, 503-505.

El-Abdellati A., Charlier J., Geldhof P., Levecke B., Demeler J., von Samson-Himmelstjerna G., Claerebout E., Vercruysse J. (2010). The use of a simplified faecal egg count reduction test for assessing anthelmintic efficacy on Belgian and German cattle farms. Veterinary Parasitology 169, 352-357.

Enøe C., Georgiadis M.P., Johnson W. O. (2000). Estimation of sensitivity and specificity of diagnostic tests and disease prevalence when the true disease state is unknown. Preventive Veterinary Medicine 45, 61-81.

Hui S. L., Zhou X. H. (1998). Evaluation of diagnostic tests without gold standards. Statistical Methods in Medical Research 7, 354-370.

Levecke B., Anderson R. M., Berkvens D., Charlier J., Devleesschauwer B., Speybroeck N., Vercruysse J., Van Aelst S. (2015). Mathematical inference on helminth egg counts in stool and its applications in mass drug administration programmes to control soil-transmitted helminthiasis in public health. Advances in Parasitology 87, 193-247.

Levecke B., De Wilde N., Vandenhoute E., Vercruysse J. (2009). Field validity and feasibility of four techniques for the detection of Trichuris in simians: a model for monitoring drug efficacy in public health? PLoS Neglected Tropical Diseases 3(1), e366.
Levecke B., Rinaldi L., Charlier J., Maurelli M.P., Morgoglione M.E., Vercruysse J., Cringoli G. (2011). Monitoring drug efficacy against gastrointestinal nematodes when faecal egg counts are low: do the analytic sensitivity and the formula matter? Parasitological Research 109, 953-957.

Morgan E.R., Cavill L., Curry G.E., Wood R.M., Mitchell E.S.E. (2005). Effects of aggregation and sample size on composite faecal egg counts in sheep. Veterinary Parasitology 131, 79-87.

Rinaldi L., Levecke B., Bosco A., Ianniello D., Pepe P., Charlier J., Cringoli G., Vercruysse J. (2014). Comparison of individual and pooled faecal samples in sheep for the assessment of gastrointestinal strongyle infection intensity and anthelmintic drug efficacy using McMaster and Mini-FLOTAC. Veterinary Parasitology 205, 216223.

Speich B., Knopp S., Mohammed K.A., Khamis I.S., RinaldiL., CringoliG., RollinsonD.,UtzingerJ.(2010). Comparative cost assessment of the Kato-Katz and FLOTAC techniques for soil-transmitted helminth diagnosis in epidemiological surveys. Parasites \& Vectors 3,71. Torgerson P. R., Paul M., Lewis F. I. (2012). The contribution of simple random sampling to observed variations in faecal egg counts. Veterinary Parasitology 188, $397-401$.

\section{ERASMUS OVER ARTSEN EN GELD (1518)}

'Ik wil toegeven dat dit beroep te edel is om tot geldopstapeling en louter gewin te dienen, zoal dit voor de alledaagse beroepen het geval is. Maar het is al te ondankbaar evenwel de passende dankbaarheid te onttrekken aan haar alleen, voor wie nooit dankbaarheid groot genoeg kan zijn. Een uitmuntend arts helpt zoals een god zonder hoop op beloning en helpt tegen de wil van de patiënten in. Maar het is snoodheid niet dankbaar te zijn voor de weldaad van een god. Hij wacht niet op het loon, maar gij verdient door de wetten gestraft te worden omdat gij uw plicht verzuimd hebt door hem niet te betalen'.

Uit: 'Een betoog over de lof van de geneeskunde' door Desiderius Erasmus van Rotterdam (oorspronkelijk ‘Encomium artis medicae', Basel 1518, vertaling L. Elaut, De Standaard, 1950) 